\title{
Clinical measures, smoking, radon exposure, and risk of lung cancer in uranium miners
}

\author{
Murray M Finkelstein
}

\begin{abstract}
Objectives-Exposure to the radioactive daughters of radon is associated with increased risk of lung cancer in mining populations. An investigation of incidence of lung cancer following a clinical survey of Ontario uranium miners was undertaken to explore whether risk associated with radon is modified by factors including smoking, radiographic silicosis, clinical symptoms, the results of lung function testing, and the temporal pattern of radon exposure.
\end{abstract}

Methods-Miners were examined in 1974 by a respiratory questionnaire, tests of lung function, and chest radiography. A random selection of $733(75 \%)$ of the original 973 participants was followed up by linkage to the Ontario Mortality and Cancer Registries.

Results-Incidence of lung cancer was increased threefold. Risk of lung cancer among miners who had stopped smoking was half that of men who continued to smoke. There was no interaction between smoking and radon exposure. Men with lung function test results consistent with airways obstruction had an increased risk of lung cancer, even after adjustment for cigarette smoking. There was no association between radiographic silicosis and risk of lung cancer. Lung cancer was associated with exposures to radon daughters accumulated in a time window four to 14 years before diagnosis, but there was little association with exposures incurred earlier than 14 years before diagnosis. Among the men diagnosed with lung cancer, the mean and median dose rates were 2.6 working level months (WLM) a year and $1.8 \mathrm{WLM}$ year in the four to 14 year exposure window.

Conclusions-Risk of lung cancer associated with radon is modified by dose and time from exposure. Risk can be substantially decreased by stopping smoking.

(Occup Environ Med 1996;53:697-702)

Ontario Ministry of

Labour, 400 University

Avenue, 7th Floor,

Toronto, Ontario, Canada

M M Finkelstein

Correspondence to: Dr Murray Finkelstein, Ontario Ministry of Labour, 400 University Avenue, 7th Floor, Toronto, Ontario, Canada M7A 1T7.

Accepted 10 May 1996
Exposure to the radioactive daughters of the gas radon is associated with increased risk of lung cancer in mining populations ${ }^{1}$ and in animals. ${ }^{2}$ In a combined analysis of 11 mining populations, risk increased with radon exposure, but for a given exposure, relative risk declined with increasing age and time since exposure. ${ }^{1}$ Data from two of those studies indicated that the risk associated with joint exposure to radon and tobacco smoke was greater than the sum of the risks associated with each factor individually. ${ }^{1}$ No clinical factors other than smoking and silicosis ${ }^{3}$ have been assessed as potential modifiers of risk of lung cancer associated with radon. Risk of lung cancer among miners may be influenced by concomitant exposures, such as silica and diesel and blasting fumes, that might damage or irritate lung tissue, potentiating the carcinogenic effect of radon. Although there is uncertainty about the ability to generalise from the results from studies of miners to residential exposures, it has been estimated that about $12 \%$ of deaths from lung cancer among residents of single family dwellings in the United States may be attributable to indoor exposure to radon. ${ }^{4}$

In 1974, a survey ${ }^{5}$ was carried out at the two uranium mines in Elliot Lake, Ontario to determine the effect of the occupational environment on the prevalence of chronic lung disease. It was the goal of the present analysis to investigate incidence of lung cancer after that survey in relation to clinical measures and occupational factors, and to explore whether risk associated with radon is modified by clinical and temporal factors.

\section{Methods}

The focus of the original survey was the prevalence of non-malignant respiratory disease in the active uranium mining population of Ontario. The roll of survey subjects was compiled from the employment records of the mining companies. Of 1043 eligible people, $967(92 \cdot 7 \%)$ volunteered to participate. Each participant was examined in 1974 by means of a modified version of the British Medical Research Council (MRC) respiratory questionnaire, tests of lung function (with a seven liter vitalograph), and chest radiography. Unfortunately, the survey records were inadvertently shredded several years before the present analysis, leaving only the computer cards that held the coded results of the survey. Some of the cards were in poor physical condition, and data could be retrieved for only 733 ( $75 \%)$ of the 973 study participants. There is evidence that the deterioration of the cards and the loss of subjects was random. Twenty three $(2.4 \%)$ of the subjects in the original survey were reported to have code 5 silicosis (explained later) and $47(4.9 \%)$ were reported 
Table 1 Mortality in the cohort from the time of examination to the end of 1992

\begin{tabular}{|c|c|c|c|}
\hline Cause of death & Observed & Expected & $S M R(95 \% C I)$ \\
\hline All causes & 117 & $108 \cdot 6$ & $1.08(0.89-1.3)$ \\
\hline All malignancies & 41 & $31 \cdot 1$ & $1.32(0.95-1.8)$ \\
\hline Digestive cancer & 9 & $9 \cdot 1$ & $0.99(0.45-1.9)$ \\
\hline Lung cancer & 26 & $11 \cdot 1$ & $2.35(1.54-3.4)$ \\
\hline Circulatory disease & 28 & 35.9 & $0.78(0.52-1.13)$ \\
\hline Respiratory disease & 8 & $5 \cdot 75$ & $1.39(0.60-2.7)$ \\
\hline Silicosis & 2 & - & - \\
\hline Violent causes & 19 & $10 \cdot 2$ & $1.86(1.12-2.9)$ \\
\hline
\end{tabular}

Table 2 Incidence of lung cancer in relation to factors and measurements in 1974

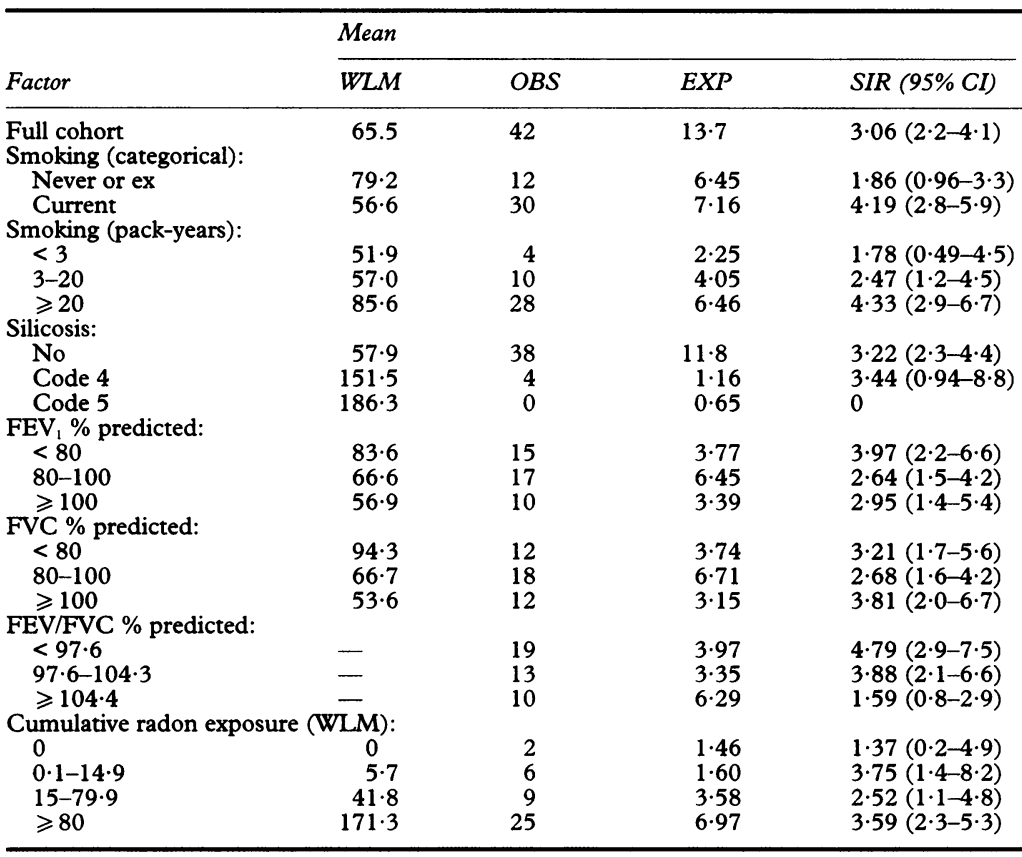

to have code 4 silicosis. ${ }^{5}$ In the data that were recovered there were 18 subjects $(2 \cdot 5 \%)$ with code 5 and $35(4 \cdot 8 \%)$ with code 4 silicosis. These proportions closely match the proportions for subjects with silicosis in the total survey population, and the participants in this study may thus be viewed as a random selection from the subjects of the original survey

The data available for each subject included current smoking status, age smoking started and age smoking stopped, average number of cigarettes a day, cough, sputum production, and wheeze; four measurements of forced vital capacity (FVC) and forced expiratory volume in one second $\left(\mathrm{FEV}_{1}\right)$; and the chest $x$ ray film diagnostic code in use in Ontario since 1929. In this diagnostic coding scheme, code 4 is possibly early silicosis and code 5 is obvious silicosis. Lung function measurements were standardised for age, height, and weight with the normal values for a non-smoking blue collar population published by National Institute for Occupational Safety and Health (NIOSH), ${ }^{6}$ with (as in the NIOSH publication) the largest of the four trials for each of FVC and FEV . Supplementary data on work history available from the Ontario miners study included an annual record of radon exposures. ${ }^{\star}$

^Exposures in the mines were measured in units of * Exposures in the mines were measured in units of working level months (WLM). One working level (WL) equals
any combination of radon progeny in one litre of air that results in the emission of $130000 \mathrm{MeV}$ of energy from $\alpha$ particles. in the emission of $130000 \mathrm{MeV}$ of energy from $\alpha$ particles.
The WLM is the product of time, in units of 170 hours, and The WLM is the product of time, in units of 170 hours, and
WL. One WLM equals a lung dose of about 0.5 rad or about 5-10 rem.
For fiscal reasons, follow up was limited to Ontario and consisted of linkage with the Ontario Mortality Database and Cancer Registry until the end of 1992. Standardised mortality (SMR) and incidence (SIR) ratios were computed with Ontario population rates and the person-years computer program. ${ }^{8}$ Proportional hazards (Cox's) regression methods ${ }^{9}$ were used to make internal comparisons of risk of lung cancer while controlling for confounding variables such as age and smoking habit. The case subjects were the 42 incident cases of lung cancer. Previous analy$\operatorname{ses}^{1}$ have shown that the dose-response relation for radon and lung cancer is effectively linear. As the Cox's model is exponential, the exposure variable was logarithmically transformed; because some subjects were unexposed, $0 \cdot 1$ working level months (WLM) was added to the exposure of each subject to avoid the infinities arising from the logarithm of 0 .

\section{Results}

\section{STANDARDISED MORTALITY AND INCIDENCE} RATIO ANALYSES

Table 1 compares mortality in the cohort with mortality in the general population of Ontario, from the time of examination in 1974 to the end of 1992 . All cause mortality was slightly increased, with the excess being attributable to the significantly increased risk of death from lung cancer and violent causes. Table 2 shows incidence of lung cancer in relation to measures obtained at the time of the survey in 1974. The first section of the table shows that incidence of lung cancer was increased threefold in the cohort in comparison with the general population. Many of these cases were diagnosed relatively late in the follow up period, and the victims were alive at the close of follow up. Incidence of cancer was not significantly increased at any other anatomical site.

\section{Cigarette smoking}

Sixteen per cent of the cohort reported that they had never smoked, and $61 \%$ were current smokers. For $96 \%$ of the cohort, data were available to compute the cumulative number of cigarettes smoked to the time of examination, assuming that the number smoked a day had been constant. Data on smoking habits subsequent to the survey were available for only a fraction of the cohort and were not used.

There were four incident lung cancers among men who reported that they had never smoked (SIR $=2 \cdot 06)$ and eight cases among former smokers $(\operatorname{SIR}=1 \cdot 78)$. Table 2 shows that risk of lung cancer among miners who were smokers at the time of the survey was twice as high as the lung cancer risk among the other men. Mean cumulative exposures to radon at the time of the survey were lower among current smokers than among the other miners. Table 2 shows a trend in lung cancer risk with increasing pack-years consumption, and in a Cox's regression model smoking was a highly significant risk factor $(P=0.0003)$. 
Chronic bronchitis

Miners were considered to have chronic bronchitis if they reported cough and sputum production for more than three months of the year. There was no association between chronic bronchitis and the number of years spent exposed to dust. In a Cox's regression model, the risk of lung cancer was increased among men with bronchitis (relative risk $2 \cdot 1$; $95 \%$ confidence interval (95\% CI) 0.99 4.63 ), but bronchitis was not an independent risk factor after accounting for smoking.

\section{Radiographic silicosis}

Fifty three subjects were diagnosed with code 4 or 5 silicosis at the time of the survey. Table 2 shows that there was no association between silicosis and the subsequent risk of lung cancer. Eighty six men were diagnosed with code 5 silicosis after the survey. In a time dependent Cox's model, which included these new cases of silicosis as they occurred, there was no association between radiographic silicosis and risk of lung cancer (relative risk $0.94 ; 95 \%$ CI $0 \cdot 48-1 \cdot 85$ ).

\section{Lung function testing}

There was no association between $\mathrm{FEV}_{1}$ and the number of years exposed to dust and a borderline association between loss of FVC and the number of years exposed to dust $(\mathbf{P}=$ $0.053)$. There was no relation between lung cancer and FVC, but the risk of lung cancer was strongly associated with decreased $\mathrm{FEV}_{1}$

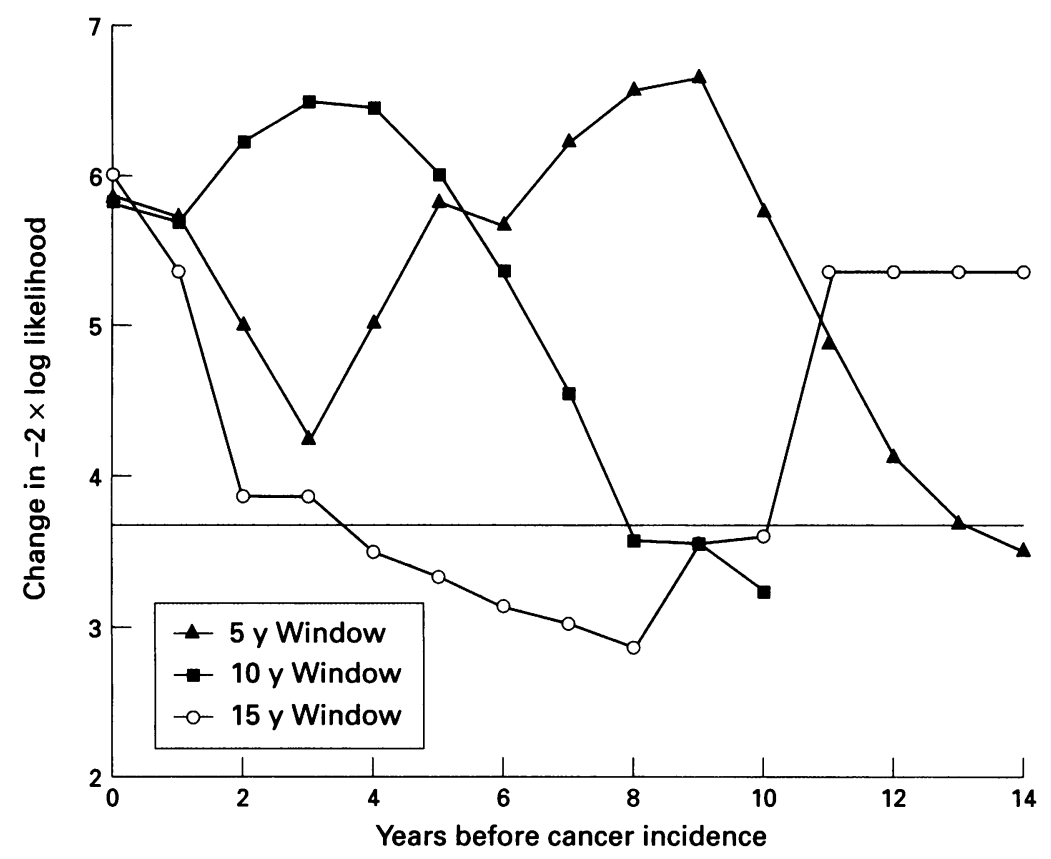

The fit of time dependent Cox's regression models to the data on incidence of lung cancer. Each point represents the fit of a model of relative risk of lung cancer, adjusted for age, in which the exposure term is the cumulative exposure to radon daughters in a time window of specified width. The horizontal axis indicates the number of years before the diagnosis of lung cancer at which the exposure window ends. Thus, for example, the leftmost point for the 10 year window represents exposures accrued from zero to 10 years before the date of diagnosis, the next point represents exposures accrued from one to 11 years before the date of diagnosis etc. The curves (which are simply line segments joining the data points) represent time windows of five, 10, and 15 years in duration. The change in log likelihood is a measure of the improvement in the fit of the model when the exposure term is added to the baseline model containing age alone. Changes in minus twice the log likelihood above the horizontal line at 3.84 indicate significant improvement in the models, but data points below 3.84 indicate that adding the exposure term did not improve the fit of the model.
$(P=0 \cdot 008)$. Among the pulmonary function variables, the relation between lung function decrement and risk of lung cancer was strongest for the ratio of $\mathrm{FEV}_{1}$ to $\mathrm{FVC}(\mathrm{P}=$ $0.002)$.

\section{Previous gold mining experience}

Kusiak and colleagues ${ }^{7}$ showed that uranium miners who had previously mined gold had a higher risk of lung cancer than uranium miners who had not. Of the 733 men in this analysis, 258 had mined gold before 1974. There was no significant difference in the lung cancer SIRs between men who had mined gold and men who had not. In a Cox's analysis, the relative risk was $1 \cdot 11(95 \%$ CI $0 \cdot 59-2 \cdot 11)$ for former gold miners.

\section{Exposure to radon}

Incidence of lung cancer was increased among exposed miners, but there was no apparent trend with exposure incurred at the time of the 1974 survey in an SIR analysis (table 2) or in a Cox's analysis $(P=0.41)$.

\section{TIME DEPENDENCY OF RADON EXPOSURE AND} RISK OF LUNG CANCER

Many subjects continued to accumulate exposure after the survey, and these data were available from the records of the study on Ontario miners to the end of 1986 . We thus investigated risk of lung cancer in the Elliot Lake cohort in relation to the time at which increments of radon dose were received, making use of the entire exposure history from 1955 to 1986 . To explore the relation between risk of lung cancer and: (a) the amount of exposure; and $(b)$ when the increment of exposure occurred, time dependent Cox's regression models were fitted to the data on incidence of lung cancer. With this analysis, the exposures of each subject diagnosed with lung cancer were compared with those of all other subjects still alive and free of disease at the date of diagnosis. If there is an association between risk of lung cancer and exposure, the exposures of subjects developing cancer will tend to be higher than the average exposures of subjects free of cancer at the date of diagnosis. For the purpose of exploration, "exposure windows" were created to include only those exposures occurring during a specified interval before the date of diagnosis of lung cancer. The exposure windows were systematically varied in width and the time between the date of diagnosis and the beginning of the exposure window.

The figure shows the fit of the time dependent Cox's regression models to the data on incidence of lung cancer. The baseline model for the relative risk of lung cancer included only an age term. The change in the statistical fit of the model (the log likelihood on the vertical axis of the figure) was examined after the addition of the radon exposure variable - the logarithm of the radon exposure-in the time window being assessed. The size of the change in $\log$ likelihood is an indication of statistical significance. Changes in minus twice the log likelihood greater than 3.84 indicate a signifi- 
Table 3 Final relative risk models fitted to the survey cohort data

\begin{tabular}{|c|c|c|c|}
\hline Term & Coefficient $(95 \%$ CI) & Pvalue & Relative risk \\
\hline \multicolumn{4}{|l|}{$\begin{array}{l}\text { (1) Exposure in 4-14 y } \\
\text { window + smoking + lung function: }\end{array}$} \\
\hline $\begin{array}{l}\text { Log age } \\
\text { Log (WLM }\end{array}$ & $\begin{array}{l}4.48(2.53-6 \cdot 46) \\
0.24(0.040-0.43)\end{array}$ & $\begin{array}{r}<0.000 \\
0.018\end{array}$ & \multirow{4}{*}{$2 \cdot 06(1 \cdot 03-4 \cdot 1)$} \\
\hline Current smoking & $0.72(0.026-1.41)$ & 0.042 & \\
\hline Pack-years & $0.012(0.0022-0.021)$ & 0.016 & \\
\hline $\begin{array}{l}\text { FEV/FVC \% predicted } \\
\text { (1A) Dose rate in } 4-14 \text { y window } \\
\text { + smoking + lung function }\end{array}$ & $-3.74(-6.30$ to -1.18$)$ & 0.004 & \\
\hline Log age & 4.53 & $<0.000$ & \multirow{5}{*}{$2 \cdot 05(1 \cdot 02-4 \cdot 1)$} \\
\hline $\log$ (dose rate WLM/y): & $0.230(0.047-0.413)$ & 0.014 & \\
\hline Current smoking & 0.72 & 0.043 & \\
\hline Pack-years & 0.011 & 0.016 & \\
\hline FEV/FVC \% predicted & $-3 \cdot 76$ & 0.004 & \\
\hline \multicolumn{4}{|l|}{$\begin{array}{l}\text { Final models (2) grouped exposure in } \\
\text { 4-14 y window }+ \text { smoking }+ \text { lung function: }\end{array}$} \\
\hline Log age & $4 \cdot 41$ & $<0.000$ & \multirow{6}{*}{$\begin{array}{l}1 \cdot 0 \\
1 \cdot 52(0 \cdot 68-3 \cdot 37) \\
2 \cdot 20(0.84-5 \cdot 75) \\
2 \cdot 10(1.05-4 \cdot 2)\end{array}$} \\
\hline $\begin{array}{l}\text { Grouped exposure WLM: } \\
0-12\end{array}$ & & 0.26 & \\
\hline $\begin{array}{l}12-25 \\
\geqslant 25\end{array}$ & & $\begin{array}{l}0 \cdot 31 \\
0 \cdot 11\end{array}$ & \\
\hline Current smoking & 0.71 & 0.043 & \\
\hline Pack-years & 0.011 & 0.017 & \\
\hline FEV/FVC \% predicted & $-3 \cdot 64$ & 0.006 & \\
\hline \multicolumn{4}{|l|}{$\begin{array}{l}\text { Model (2A) grouped dose rate in } \\
4-14 \text { y window + smoking + lung function: }\end{array}$} \\
\hline Log age & $4 \cdot 71$ & $<0.000$ & \multirow{6}{*}{$\begin{array}{l}1.0 \\
2 \cdot 69(1.09-6.68) \\
3.05(1.23-7.59 \\
2.08(1.04-4.2)\end{array}$} \\
\hline $\begin{array}{l}\text { Grouped dose rate WLM/y: } \\
0-0.99\end{array}$ & & 0.042 & \\
\hline $\begin{array}{l}1-1 \cdot 99 \\
\geqslant 2\end{array}$ & & $\begin{array}{l}0.033 \\
0.016\end{array}$ & \\
\hline Current smoking & 0.73 & 0.040 & \\
\hline Pack-years & 0.011 & 0.020 & \\
\hline FEV/FVC $\%$ predicted & $-3 \cdot 70$ & 0.005 & \\
\hline
\end{tabular}

cant improvement in the fit to the data when the exposure term is added to the model that contained age alone. The three curves (figure) represent exposures accumulated in windows of five, 10, and 15 years duration. The end dates of the exposure windows were lagged zero to 10 years before the date of diagnosis of lung cancer, as indicated on the horizontal axis. The best fits to the data, as indicated by the largest change in log likelihood, occurred for exposures ending eight or nine years before diagnosis in the exposure windows of five years width, or for exposures ending three or four years before diagnosis in the exposure windows of 10 years of width. Exposures incurred earlier than 14 years before the date of diagnosis had little association with risk of lung cancer, even though they were generally larger in magnitude than the more recent exposures. The mean cumulative exposure among the cases earlier than 14 years before diagnosis was 81.4 (median 67.2) WLM. The mean exposure among the cases in the time window four-14 years before diagnosis-was $25 \cdot 6$ (median 15.2) WLM.

\section{MULTIVARIATE ANALYSES}

It has become customary ${ }^{1}$ in the analysis of populations exposed to radon to use time windows of five to 14 , and $\geqslant 15$ years before death. In the present study, the interval four to 14 years before diagnosis was selected for the most recent time window because incidence of cancer was used as the end point. The approach to analysis was to start with a time dependent Cox's model which contained only age as an explanatory variable for risk of lung cancer. New models were formed by adding additional explanatory variables and interaction terms. The additional terms were retained only if they made significant improvements to the fit of the models, or were important con- founders, as indicated by changes in the radon exposure coefficient. Table 3 shows the results.

Age is a strong risk factor for lung cancer and risk varied with the fourth or fifth power of age in this cohort. This is consistent with findings in cohorts of smokers. ${ }^{10}$ Adding exposure in the four to 14 year time window provided a significant improvement to the fit of the model containing age alone $(P=0.029)$. An interaction between exposure and age was not significant $(P=0.63)$, although the sign of the coefficient was negative, suggesting decreasing relative risk with increasing age. Exposures earlier than 15 years before the date of diagnosis made no significant contribution to risk of lung cancer.

Current smoking status and the number of cigarettes smoked to the time of the survey were each significantly associated with risk of lung cancer. The coefficient for the radon term changed only marginally when smoking was added, indicating no confounding of the risk attributable to radon by smoking. An interaction between smoking and radon was not significant, indicating that a multiplicative smoking-radon exposure model was compatible with the data. The $\mathrm{FEV}_{1} / \mathrm{FVC} \%$ predicted lung function term was highly significant and the coefficient for exposure to radon was unchanged by the addition of the new variable.

As workplace exposure standards are usually expressed in terms of dose rate, we computed the dose rate for the members of the cohort as the sum of the exposures in the four to 14 year window divided by the number of months worked during that window. Among the men diagnosed with lung cancer, the mean and median exposures were $25 \cdot 6$ and $15 \cdot 2$ WLM in the four to 14 year dose window, and the mean and median dose rates were 2.6 WLM/year and $1.8 \mathrm{WLM} /$ year. Dose rate in 
the four to 14 year exposure window was significantly associated with risk of lung cancer, but exposures incurred 15 or more years before diagnosis were not associated with risk of lung cancer.

\section{Discussion}

Follow up of uranium miners participating in a 1974 survey has replicated the findings of previous studies, and has also produced some new information. In comparisons with the population of Ontario, lung cancer mortalities and incidences were $2 \cdot 4$-fold and threefold higher than expected. Because follow up during the period 1987-92 was limited to Ontario, it is possible that some cases of lung cancer were missed, leading to underestimates of the lung cancer mortality and incidences in the cohort.

Data on smoking were obtained by questionnaire and are of uncertain accuracy with respect to smoking status and estimates of pack-years smoked. It is probable that the status of men who were smokers at the time of the survey has been correctly ascertained, but there may be some misclassification of men who reported that they had stopped smoking or never smoked. There was no difference in risk of lung cancer between men who had never smoked and men who had stopped. One would have expected that former smokers would have a higher risk than never smokers. The absence of a difference may reflect low statistical power, but might be attributable to misclassification. There was no information about changes in smoking habit after the survey, and this is a potential source of misclassification. Risk of lung cancer among miners who had stopped smoking was only half that of men who continued to smoke. Smoking was not an effect modifier for radon-that is, the relative risk due to radon was independent of smoking history.

Men with lung function results consistent with airways obstruction had an increased risk of lung cancer. This risk factor remained in the models even after adjustment for cigarette smoking, an observation made previously in community studies of smoking and risk of lung cancer. ${ }^{112}$ It is not clear whether airways obstruction plays a physiological part, perhaps by impeding the clearance of carcinogens, or whether including lung function in statistical models corrects misclassification of smoking histories. There was no association between radiographic silicosis and risk of lung cancer. We have no explanation for the discrepancy with the results of previous studies in Ontario. ${ }^{13}$

Risk of lung cancer was associated with exposure to radon accumulated in a time window four to 14 years before diagnosis. There was little association with exposures accrued earlier than this. Previous studies have found that risk declined with time since exposure ${ }^{1}$; in some of them, risk was still present for exposures incurred earlier than 15 years before death. The present cohort may have been too small to detect the lower risk attributable to these earlier exposures. The mechanism of radon carcinogenesis is uncertain. Current models of cancer development begin with induced mutations of DNA (initiating events) followed by a series of additional mutations leading ultimately to unrestrained cellular growth. Cell multiplication caused by irritants such as cigarette smoke increases the pool of "initiated cells" at risk of further mutational events. It is not clear where radon decay energy acts in this chain. The lack of importance of exposures distant in time suggests either that cells carrying mutational events caused by early exposures are lost, or that mutational events caused by radon are important late in the pathway leading to cancer.

The observation of lower or absent risk attributable to remote exposures has important implications for occupational and environmental standards for radon. Because of improved conditions in the mines, radon exposures in the four to 14 year time window tended to be much lower than the averages seen in previous studies and were generally close to the current occupational standard of 4 WLM per year. Among the men diagnosed with lung cancer in the survey cohort, the mean and median cumulative exposures were 25.6 and 15.2 WLM in the four to 14 year dose window, and the mean and median dose rates were $2.6 \mathrm{WLM} /$ year and $1.8 \mathrm{WLM} /$ year in that window. The important exposures in this cohort seem to be in the region of the current occupational exposure limit.

Several weaknesses should be mentioned. The population size is smaller than in other cohorts, leading to wide confidence intervals, but the effects observed are so strong that most risk factors reach significance. For fiscal reasons, follow up was limited to Ontario. As a result, some deaths and diagnoses from lung cancer were probably missed, leading to underestimates of the standardised mortality and incidence ratios. The effect on the Cox's regression coefficients depends on how the exposures of the men omitted compare with the exposures of the other members of the cohort. Some simulation trials suggested that the effect of underestimates is not likely to be large. The small number of events also influences the precision of estimates of the risk per unit exposure and the identification of the effective time window. Misclassification of individual radon exposure estimates would be anticipated to reduce estimates of the doseresponse effect.

Limited data were available for risk factors of men after the survey. All analyses were thus carried out with data on smoking from the 1974 questionnaire. It is likely that the habits of some men changed after the survey. It is difficult to predict how these changes might affect the risk estimates, as some men might have stopped smoking whereas others started or restarted. The approximate nature of the data on smoking also adds uncertainty to the investigation of effect modification of radon by smoking. Longitudinal data were available for silicosis, and were used in the analyses of that risk factor. 
Workers exposed to radon in underground mines are at increased risk of lung cancer as a result of their exposures to the radioactive decay products of radon and its daughters. There is, at the moment, no medical intervention which can decrease the risk. Screening with chest radiographs or sputum cytology has not been shown to decrease mortality from lung cancer. ${ }^{14}$ It is well substantiated that former smokers are at lower risk of lung cancers than smokers who persist in the habit. ${ }^{15}$ This behavioural change on the part of men exposed to the respiratory carcinogen, radon, can substantially modify their risk of lung cancer. It is thus recommended that men exposed to workplace carcinogens be urged to stop smoking.

Robert Kusiak provided exposure data collected for the Ontario miners study. Linkage of the cohort roll to the Ontario Mortality Database and Cancer Registry was performed by staff of the Ontario Cancer Treatment and Research Foundation.

1 Lubin JH, Boice JD, Edling C, Hornung RW, Howe G, Kumz E, et al. Radon and lung cancer risk: $a$ joint analysis of 11 underground miners studies. Bethesda: National Institutes of Health, 1994.

2 Cross FT. Invited commentary: residential radon risks from the perspective of experimental animal studies. $A m \mathcal{F}$ Epidemiol 1994;140:333-9.
3 Finkelstein M. Radiographic abnormalities and the risk of lung cancer among workers exposed to silica dust in Ontario. Can Med Assoc $\mathcal{F}$ 1995;152:37-43.

4 Lubin $\mathrm{JH}$. Invited commentary: lung cancer and exposure to residential radon. $A m \mathcal{F}$ Epidemiol 1994;140:323-32.

5 Ontario Ministry of Health. Survey of certain conditions of the respiratory organs among persons employed underground and in surface crushers and mills of two operating uranium mines a Elliot Lake, Ontario. Toronto: Ontario Ministry of Health. 1975.

6 Petersen M, Hankinson J. Spirometry reference values for nonexposed blue-collar workers. F Occup Med 1985;27: 644-50.

7 Kusiak RA, Ritchie AC, Muller J, Springer J. Mortality from lung cancer in Ontario uranium miners. $B r \mathcal{F}$ Ind Med 1993;50:920-8.

8 Coleman M, Douglas A, Hermon C, Peto J. Cohort study analysis with a fortran computer program. Int $\mathcal{f}$ Epidemiol 1986;15:134-7.

9 SPSS. SPSS for Windows advanced statistics release $6 \cdot 1$. Chicago: SPSS, 1994.

10 Lubin JH, Blot WJ. Lung cancer and smoking cessation patterns of risk. $\mathcal{F}$ Natl Cancer Inst 1993;85:422-3.

11 Nomura A, Stemmermann GN, Chyou PH, Marcus EB, Buist AS. Prospective study of pulmonary function and lung cancer. Am Rev Respir Dis 1991;144:307-11.

12 Kuller LH, Ockene J, Meilahn E, Svendsen KH. Relation of forced expiratory volume in one second $\left(\mathrm{FEV}_{1}\right)$ to lung cancer mortality in the multiple risk factor intervention cancer mortality in the multiple risk factor interven
trial (MRFTT). Am $f$ Epidemiol 1990;132:265-74.

13 Finkelstein $M$. Radiographic abnormalities and the risk of lung cancer among workers exposed to silica dust in Ontario. Can Med Assoc ₹ 1995;152:37-43

14 Kubik A, Parkin DM, Khlat M, Erban J, Polak J, Ademec $M$. Lack of benefit from semi-annual screening for cancer of the lung: follow-up report of a randomized controlled trial on a population of high-risk males in Czechoslovakia. Int $f$ Cancer 1990;45:26-33.

15 Doll $R$, Peto $R$, Wheatley K, Gray R, Sutherland I. Mortality in relation to smoking: 40 years' observations on male British doctors. BM于 1994;309:901-11. 